\title{
Spontaneous Olfactory Receptor Neuron Activity Determines Follower Cell Response Properties
}

\author{
Joby Joseph, Felice A. Dunn, and Mark Stopfer \\ National Institutes of Health, National Institute of Child Health and Human Development, Bethesda, Maryland 20892
}

\begin{abstract}
Noisy or spontaneous activity is common in neural systems and poses a challenge to detecting and discriminating signals. Here we use the locust to answer fundamental questions about noise in the olfactory system: Where does spontaneous activity originate? How is this activity propagated or reduced throughout multiple stages of neural processing? What mechanisms favor the detection of signals despite the presence of spontaneous activity? We found that spontaneous activity long observed in the secondary projection neurons (PNs) originates almost entirely from the primary olfactory receptor neurons (ORNs) rather than from spontaneous circuit interactions in the antennal lobe, and that spontaneous activity in ORNs tonically depolarizes the resting membrane potentials of their target PNs and local neurons (LNs) and indirectly tonically depolarizes tertiary Kenyon cells (KCs). However, because these neurons have different response thresholds, in the absence of odor stimulation, ORNs and PNs display a high spontaneous firing rate but KCs are nearly silent. Finally, we used a simulation of the olfactory network to show that discrimination of signal and noise in the KCs is best when threshold levels are set so that baseline activity in PNs persists. Our results show how the olfactory system benefits from making a signal detection decision after a point of maximal information convergence, e.g., after KCs pool inputs from many PNs.
\end{abstract}

\section{Introduction}

Neural activity in the absence of obvious stimuli appears throughout the central and peripheral nervous systems (Rieke and Baylor, 2000; Maye et al., 2007; Kenet et al., 2003; MacLean et al., 2005; Tritsch et al., 2007). Such spontaneous activity may play useful roles (Limb and Braun, 2008). During development, for example, spontaneous activity is required to form appropriate connections in the auditory system (Tritsch et al., 2007) and to maintain newly learned material (Dave and Margoliash, 2000; Euston et al., 2007). In the olfactory system of vertebrates (but not Drosophila; Jefferis et al., 2004), spontaneous activity is required for the development and maintenance of glomerular maps (Yu et al., 2004). In other cases, though, spontaneous activity may not benefit the organism at all but may rather limit perception and behavior.

We used the locust olfactory system to investigate spontaneous activity at multiple points along a sensory pathway. In insects, olfactory receptor neurons (ORNs) are distributed along the antenna. The ORNs project to the antennal lobe (AL, a brain structure analogous to the olfactory bulb of vertebrates) and synapse

Received Aug. 15, 2011; revised Dec. 30, 2011; accepted Jan. 10, 2012.

Author contributions: J.J., F.A.D., and M.S. designed research; J.J. and F.A.D. performed research; J.J. and F.A.D. analyzed data; J.J., F.A.D., and M.S. wrote the paper.

This work was supported by an intramural award from NICHD to M.S. We are grateful to: Tom Talbot, Gary Melvin, and George Dold of the NIH Section on Instrumentation Core Facility for designing and constructing the antenna cooling device; to members of the Stopfer Laboratory and Zhishang Zhou, Greg Field and Gabe Murphy for helpful comments on the manuscript; and to Kui Sun for excellent animal care.

Correspondence should be addressed to Mark Stopfer, NIH-NICHD, Building 35, 35 Lincoln Drive, Room 3A-102, MSC 3715, Bethesda, MD 20892, E-mail: stopferm@mail.nih.gov.

J. Joseph's present address: Center for Neural and Cognitive Sciences, University of Hyderabad, India.

F. A. Dunn's present address: University of Washington, Department of Biological Structure, Seattle, WA 98195.

DOI:10.1523/JNEUROSCI.4207-11.2012

Copyright $\odot 2012$ the authors $\quad 0270-6474 / 12 / 322900-11 \$ 15.00 / 0$ there upon mainly inhibitory local neurons (LNs) and excitatory projection neurons (PNs). The PNs, in turn, send processes to the Kenyon cells (KCs) in the mushroom body (MB), a structure analogous to the vertebrate olfactory cortex. Our recordings revealed high levels of spontaneous activity in the ORNs and PNs, but very little in $\mathrm{KCs}$.

Where does this spontaneous activity originate? How does spontaneous activity propagate from one population of neurons to the next, and how and why is it sharply reduced in neurons two synapses removed from the ORNs? And what effects does spontaneous activity exert upon olfactory coding?

The locust offers an anatomical advantage for such work: their ORNs are easily accessible for study and are distant from the first olfactory neuropil. We found that reversibly silencing the ORNs by cooling the antenna significantly hyperpolarized the resting membrane potentials and reduced spiking of PNs, LNs, and KCs. Our recordings show that spontaneous activity originates in the ORNs and exerts a constant influence upon the follower cells.

Sparse codes, characterized by few spikes in few neurons, offer numerous advantages for representing sensory stimuli (Young and Yamane, 1992; Hahnloser et al., 2002; DeWeese et al., 2003; Brecht et al., 2004). To explore why spontaneous activity originating in ORNs is passed to vigorously spiking secondary neurons but is then sharply curtailed in nearly silent tertiary neurons, we simulated the success of KCs in detecting signal within noise given varying degrees of input convergence, ORN signal strength, and firing thresholds. Our simulations showed that, under challenging conditions, signal-to-noise detection in KCs is best when response thresholds are relatively low in $\mathrm{PNs}$; this configuration allows for the maximal convergence of information in KCs. Our results in the locust demonstrate a general strategy for how circuitry with highly sensitive (and therefore noisy) receptors in the 
periphery and patterns of convergence may set thresholds for optimal discrimination of signal from noise and for coding the results sparsely.

\section{Materials and Methods}

Animal preparation. Locusts were raised in our crowded colony. Young male or female adults were restrained with a wax, saline-filled chamber built up around the head capsule. The brain was exposed, desheathed, and bathed in locust saline, as described previously (Stopfer et al., 2003; Laurent and Davidowitz, 1994). One antenna was threaded out of the wax chamber through tubing, leaving all but a few millimeters of the antenna exposed to the air.

Odor delivery and air flow. Odorant timing and pressure was controlled by a computer-regulated pneumatic Picopump as described previously (Laurent and Naraghi, 1994). Briefly, odorant solution (hexanol, $20 \mathrm{ml}$ ) was placed in a $60 \mathrm{ml}$ glass bottle. Odor pulses were delivered by injecting a measured volume $(0.1 \mathrm{~L} / \mathrm{min})$ of the static headspace above the odorants into an activated carbon-filtered and dehydrated air stream $(0.75$ $\mathrm{L} / \mathrm{min}$ ) flowing continuously across the antenna. A large $(11 \mathrm{~cm})$ vacuum funnel was placed behind the antenna to remove the delivered odorants.

Using a custom-built Peltier device, we cooled the antenna by varying the temperature, between $25^{\circ} \mathrm{C}$ and $4^{\circ} \mathrm{C}$, of the constant air flow. To thermally insulate the brain from chilled air, the antenna was threaded through a small hole in a plastic barrier and sealed in place with a mixture of mineral oil and Vaseline. Separate digital thermometers monitored the temperature of the saline bathing the brain and the temperature of the antenna. Despite the thermal barrier, we found that the temperature of the saline bathing the brain dropped by $\sim 2^{\circ} \mathrm{C}$ when the antenna was cooled. Because temperature shifts can affect the membrane potentials of neurons (Griffin and Boulant, 1995), we tested for the effects of directly cooling the brain by $\sim 2^{\circ} \mathrm{C}$ by adding drops of chilled saline directly to the bath. For one experiment we isolated the antenna from environmental odorants by coating it with a mixture of mineral oil and Vaseline and later removed the antenna, cutting it as close to its base as possible (likely leaving intact a few sensilla proximal to the thermal barrier).

Extracellular $P N$ and LFP recordings. We recorded local field potentials from the calyx of the MB using custom twisted gold plated nichrome wire metal tetrodes and amplified them with a custom amplifier. We made extracellular recordings from PNs, the only spiking neurons in the locust AL (Laurent and Naraghi, 1994), using silicon tetrode probes from the Center for Neural Communication Technology (University of Michigan, Ann Arbor, MI; Drake et al., 1988). We sorted PN spikes offline using the Spike-o-Matic algorithm (Pouzat et al., 2002) implemented in Igor Pro (WaveMetrics). Records from unambiguously separated clusters (see quantitative criteria in Pouzat et al., 2002) were used for analysis. Results were further analyzed by custom programs written in MATLAB (The MathWorks) and Igor Pro software.

Extracellular sensilla recordings. Intact locusts were taped firmly to a glass platform and one antenna was fixed to the platform with thin strips of tape. Sensillae were visualized under $160 \times$ magnification. Recordings were made from the base of the sensillae with glass pipettes pulled to $\sim 40$ $\mathrm{M} \Omega$, filled with locust saline, and threaded with silver-silver chloride wire. Signals were amplified using a Grass P55amplifier (Grass Technologies) with 10,000× gain and filtered between $300 \mathrm{~Hz}$ and $6 \mathrm{kHz}$.

Patch-clamp recordings. We recorded from PNs, LNs, and KCs by whole-cell current-clamp. The internal solution contained $140 \mathrm{~mm}$ K-aspartate, 10 mм HEPES, 1 mм KCl, 4 mм Mg-ATP, $0.5 \mathrm{~mm} \mathrm{Na}_{3} \mathrm{GTP}$, and 1 mm EGTA (Wilson et al., 2004), pH was adjusted to 7.2 with $\mathrm{K}-\mathrm{OH}$, and osmolarity was increased to $330 \mathrm{mOsm}$ to match approximately that of the external solution. Values shown in figures and text were corrected for the junction potential of $17.2 \mathrm{mV}$. Voltages were low-pass filtered at 4 $\mathrm{kHz}$; data were acquired at $10 \mathrm{kHz}$. Individual traces shown in Figures 3-5 were low-pass filtered at $100 \mathrm{~Hz}$. The locust brain was illuminated with a light source external to the microscope and visualized with a camera above a $40 \times$ immersion objective. LNs were identified by their large, oblong somata, clustering within the medial AL, and by their inability to generate sodium spikes upon current injection. PNs displayed a steady firing rate of sodium spikes in the absence of stimulation. We targeted KCs near the medial edge of the MB. To calculate the resting membrane potential of each cell type, we clipped the sodium spikes and averaged the voltages in the $2 \mathrm{~s}$ preceding the odor stimulation (see Figs. $3-5 A-C)$.

Data analysis. Analysis other than spike sorting was performed in MATLAB. Average firing responses of PNs were calculated over $2 \mathrm{~s}$ windows; for trials including odor presentations, these windows began with the odor delivery. Statistical comparisons of results obtained through different antenna treatments shown in Figure 2 were made by two-way ANOVAs followed by Bonferroni-corrected multiple comparisons tests with significance level set to 0.05 . For comparisons shown in Figures 3-5, statistical significance was determined using two sample $t$ tests. Overall, data points for the change in membrane potential at the lowest and highest antenna temperatures were significantly different $(p<0.001)$; data points for the change in membrane potential at the highest antenna temperature compared to that of the lowest control saline temperature were not significantly different. The data points for the change in membrane potential at the lowest antenna temperature compared to that of the lowest control saline temperature were significantly different $(p<0.001)$.

Simulations. To explore the significance of olfactory circuit parameters for accurate odor detection by KCs, we wrote a program in MATLAB to model ORNs, PNs, and KCs in which the amount of signal present in noisy ORNs, the convergence patterns of ORNs, PNs, and KCs, and the firing thresholds of PNs and KCs could be varied.

Model ORNs, consistent with our recordings made in vivo, were given a mean background firing rate of $\lambda=5$ spikes/s, and responses to odor were simulated with transient increases in the spike rate by $\delta \mathrm{Hz}$ (see Fig. $7)$. Odor trials and no-odor trials were simulated with equal probability. In the no-odor case, all ORNs fired with baseline probability $(\lambda)$. In the odor case, all ORNs provided this baseline of activity plus a small uniform increase in firing rate $(\lambda+\delta)$. For each trial, numbers of spikes, $k$, in each step were drawn from a Poisson distribution $(p(k)=$ $\left.\left(\lambda^{k} \exp (-\lambda)\right) / k !\right)$ around the mean of $\lambda$. Parameters of our simulation were drawn from our experimental results.

All synapses from ORNs to PNs were equally weighted so that, at each time step, input to a PN, $y$, was the mean of the spike counts from $N$ ORNs converging to the PN: $y=(1 / N) \Sigma_{\mathrm{i}} k_{\mathrm{i}}$, with $N$ set to $5,10,50$, or 100 ORNs for each PN in separate simulations (see Fig. 7). A PN's response at any instant was Poisson distributed with a mean proportional to its synaptic input exceeding its firing threshold ( $\lambda=y-$ Threshold $)$.

Similarly, at each time step input to a KC consisted of the mean output of PNs converging upon that KC (450 PNs/KC; Jortner et al., 2007). A KC was said to fire when its mean input exceeded its firing threshold. We varied the firing thresholds of PNs ( 80 steps from 0 to 8 ; the abscissa in Figs. $6 \mathrm{D}$ and 7 ) and of KCs [ 100 steps from 0 to 10; e.g., along the receiver operating characteristic (ROC) curve in Fig. 6C].

To determine the signal detection performance of a $\mathrm{KC}$ under each test condition we constructed a set of standard ROC curves. We scored true and false positives when the input to a $\mathrm{KC}$ exceeded its threshold: true when such inputs occurred during an odor presentation; false when such inputs occurred during a no-odor presentation. The percentages of true and false positives were calculated from 1500 trials. We defined the quality of an optimal detector as the maximum distance of points on the ROC curve from the diagonal (representing chance), as shown in Figure 6C. For each level of firing threshold in PNs we then determined the PNs' baseline firing rate and the rate corresponding to the KC's optimal detection (Fig. 6D).

\section{Results}

Baseline spiking in PNs is caused by the tonic spiking of ORNs Where does spontaneous activity arise? Our recordings from locusts showed that ORNs and PNs spiked tonically even in the absence of odorant delivery, suggesting spontaneous activity arises from early stages of olfactory processing. Unlike the ORNs and PNs, the KCs were nearly silent unless activated by an odor puff (Fig. 1A; Laurent and Naraghi, 1994; Perez-Orive et al., 2004). We found we could reversibly silence the ORNs by placing the antenna in a stream of chilled air. A thermal barrier, through 
A

ORNs

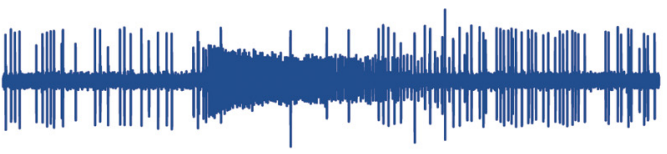

PN

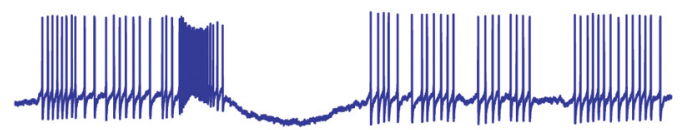

KC

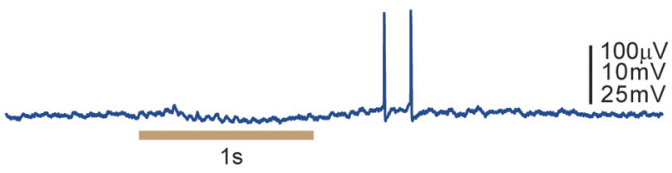

C

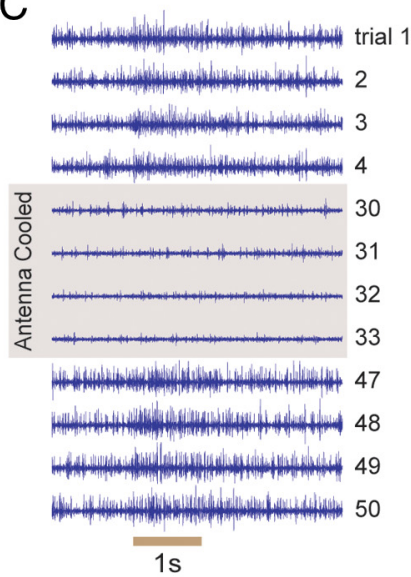

D

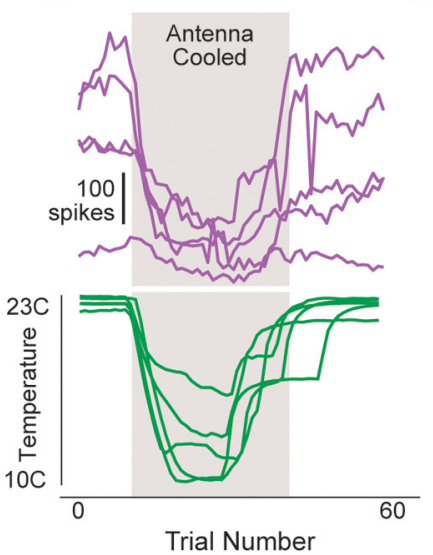

E
B
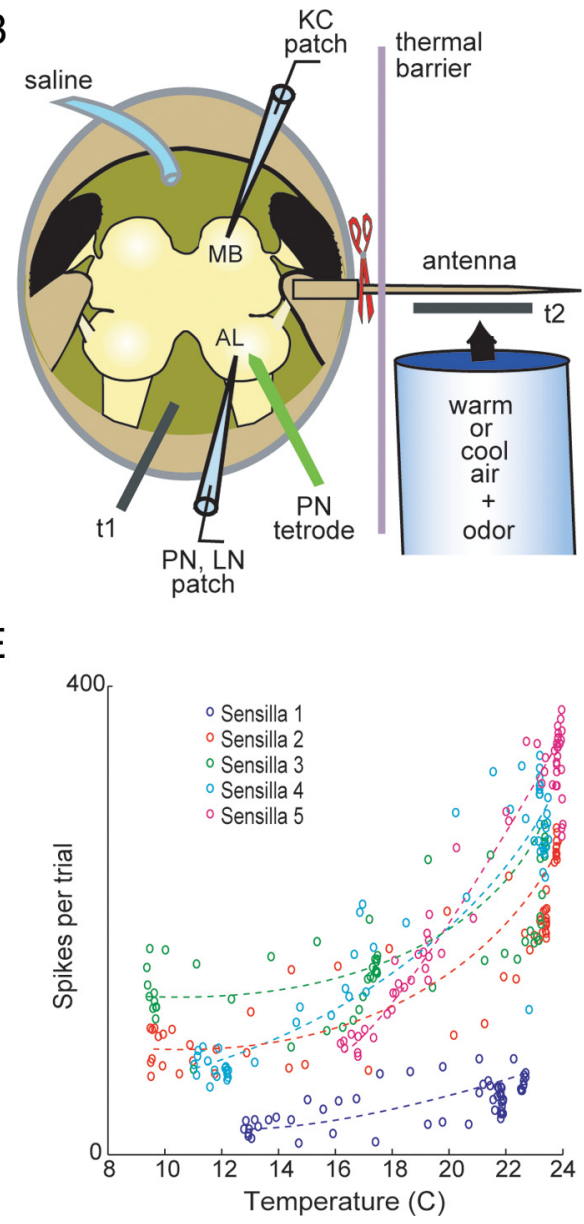

Figure 1. Spontaneous and odor-evoked spiking in olfactory neurons are nearly abolished by cooling the antenna. $A, 0$ lfactory receptor neurons, 0 RNs, and projection neurons, PNs, exhibit higher baseline activity than the Kenyon cells, KCs $(\sim 5, \sim 2.5$, and $\sim 0$ spikes/s, respectively). Examples show responses to an odor presentation (horizontal tan bar) in ORNs recorded extracellularly from a sensillum (each of which contains multiple ORNs, whose spikes can be sorted by size and shape); a PN, recorded intracellularly; and a KC, recorded intracellularly. $\boldsymbol{B}$, Diagram of the recording configuration and apparatus to manipulate antenna function. Temperature of the airflow over the antenna was regulated and recorded by thermometers in the bath near the brain (t1) and in the air adjacent to the antenna ( $\mathrm{t} 2)$. A thermal barrier prevented chilled air from cooling the brain. We used tetrodes to make extracellular recordings of spikes from PNs in the antennal lobe, AL, and in separate experiments, glass patch electrodes to make whole-cell recordings from PNs, LNs, and, in the mushroom bodies (MBs), from KCs. C, Example sensilla recording shows that the firing of ORNs decreased when the antenna was cooled (shaded background) but resumed when the antenna was returned to room temperature. $D$, Spikes per trial for all ORNs (purple) from 5 sensilla recordings as antenna temperature (green) was varied. E, Scatter plot shows all tested sensilla produced fewer spikes when cooled. Curves were drawn by eye. For most experiments, antenna temperatures were reduced below the levels shown here; large changes in temperature caused both the electrode and sensillum to change shape, making it difficult to maintain recordings from ORNs across the full range of temperatures.

which we threaded the antenna, prevented chilled air from reaching the animal's head (Fig. $1 B$ ). Figure $1 C$ shows an example of a sensilla recording in which baseline and odor-elicited spiking was nearly eliminated by cooling the antenna. Results from several such experiments are summarized in Figure 1,D and $E$. We used this technique to test the impact of activity in ORNs on the responses of downstream neurons.

To test the origins of spontaneous activity, we subjected the intact animal's antenna to a sequence of treatments while making recordings with tetrodes from PNs. These treatments are numbered 1-5 and are keyed to a gray scale in Figure 2. (1) First, we recorded spontaneous activity in PNs under control conditions; then we reversibly altered activity in the ORNs by (2) cooling and then (3) rewarming the antenna; then (4) we irreversibly isolated the antenna from the environment by covering all exposed sensilla with a viscous barrier (mixture of Vaseline and mineral oil); and finally (5), we removed the antenna by cutting through its base. Throughout these treatments we monitored the spike rates of the PNs, the temperature next to the antenna, and the temperature of the saline bathing the brain.
Figure 2 illustrates the spiking of PNs during the sequence of treatments. At first (1), an example PN (Fig. 2A) displayed a typical amount of spontaneous spiking (shown as rasters; Mazor and Laurent, 2005) before and after a puff of odor (vertical gray bar at 2-3 s). PNs characteristically respond to odors with sequences of excitation and inhibition (Laurent and Davidowitz, 1994). Responding to the odor puff, this example PN was first inhibited, then fired a burst of spikes, and then was again briefly inhibited before returning to a background level of spiking. When we gradually cooled the antenna (2), baseline and odorevoked spiking in the PN nearly ceased. In addition, the temporal structure of the PN's response to odor (the successive epochs of inhibition, excitation, and inhibition) gradually changed with the temperature of the antenna (see Fig. 8). Background and odorelicited spiking returned to the baseline level as the antenna was warmed back to the control temperature (3). Covering the antenna (4) eliminated the $\mathrm{PN}^{\prime}$ s responses to odorants. However, baseline spiking was barely affected, indicating that spontaneous activity in ORNs can arise within the sensilla even when environmental odors were prevented from reaching the antenna. Finally, 

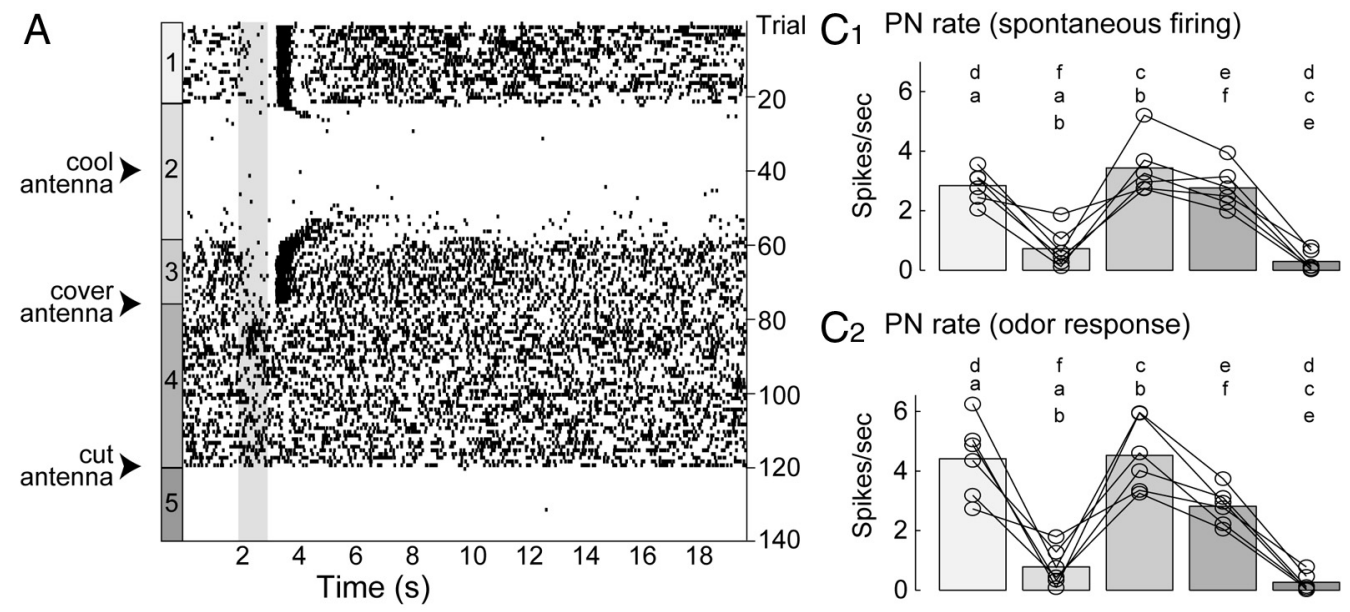

$\mathrm{C}_{2} \mathrm{PN}$ rate (odor response)

B

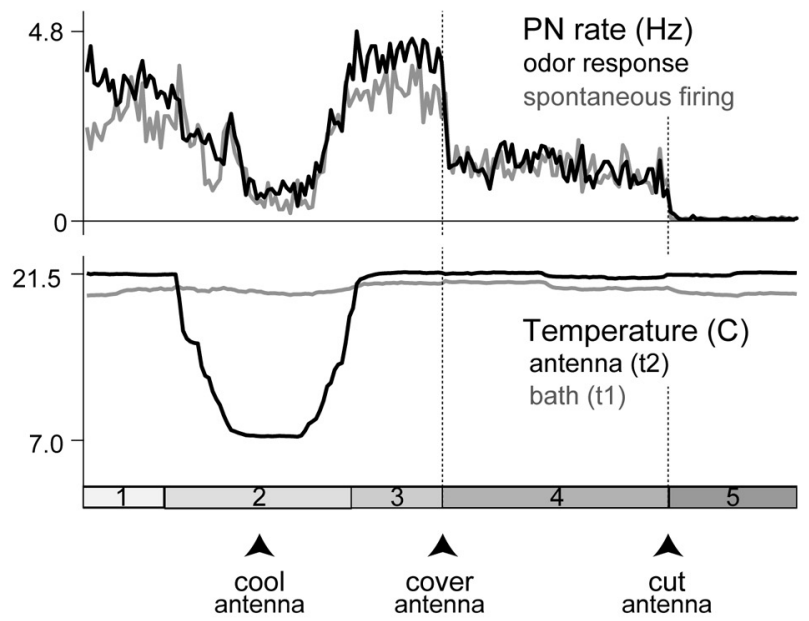

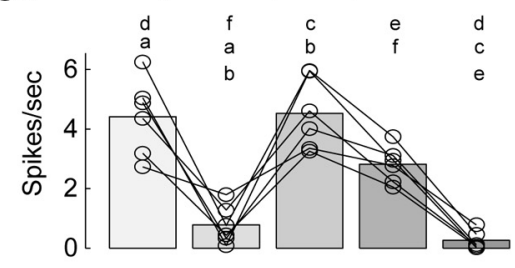

C3 Antenna temperature

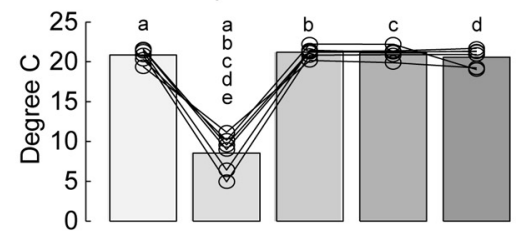

$\mathrm{C}_{4}$ Bath temperature

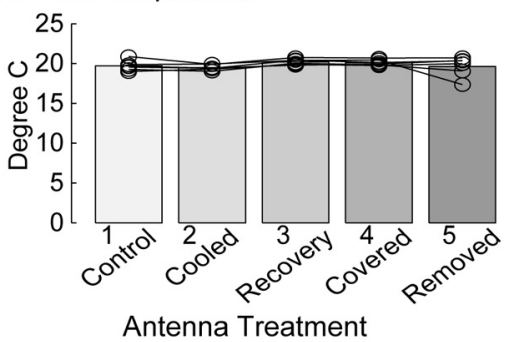

Figure 2. Cooling and removing the antenna had similar effects on projection neurons. $A$, An example PN recorded during a sequence of manipulations to the antenna; each horizontal raster sweep indicates spike times during a $20 \mathrm{~s}$ trial; vertical gray bar at $2-3 \mathrm{~s}$ indicates odor presentation; numbered gray bars at left indicate antenna treatments: (1) recordings began at room temperature (top); (2) the antenna was then cooled; (3) returned to room temperature; (4) covered (with Vaseline and mineral oil) to isolate the antenna from environmental odors; and then (5) the antenna was finally removed. $\boldsymbol{B}$, Summary of responses in the example PN shown in $A$ : manipulations to the antenna affected spiking in the PN before (gray line; spike rates calculated over the $0-2$ s in $A$ ) and during the odor stimulus (black line; $2-4$ s). Note that the response of the PN to the odor, characteristically, included both periods of excitation and inhibition, so overall, the odor increased spiking only slightly. Temperatures near the brain (t1, gray line) and the antenna (t2, black line) are shown below. Numbered gray bars at bottom match numbered vertical bars in $A$. C, Summary results from 21 PNs (6 locusts) show responses to antenna manipulations named at the bottom of $C_{4}$. Circles: average result from each locust; bars: average across locusts. Different letters (a-f) indicate results significantly different from each other (multiple comparisons tests following two-way ANOVA and Bonferroni correction, $p<0.001$ ).

removing the antenna (5), like cooling the ORNs, completely silenced the PN, suggesting that its baseline activity is inherited entirely from the ORNs. Figure $2 B$ summarizes the results of this typical experiment. Results from six locusts (21 PNs) are summarized in Figure $2 C$; circles and lines indicate results from each animal; gray bars indicate their means. Note in Figure $2 C_{1,2}$ that odor presentations raised the average firing rates of PNs only slightly because odors generally elicit responses containing both excitatory and inhibitory components (see, for example, the PN in Fig. 1A; Laurent and Davidowitz, 1994; Mazor and Laurent, 2005). During the cooling phase, the temperature near the antenna decreased to a mean of $8.5^{\circ} \mathrm{C}$ (Fig. $2 C_{3}$ ), yet the temperature inside the head capsule remained nearly constant $\left(21.5^{\circ} \mathrm{C}\right)$ throughout these experiments (Fig. $2 C_{4}$ ).

Baseline input from the ORNs tonically depolarizes PNs, LNs, and KCs

What effect does the ongoing barrage of activity in ORNs have upon follower neurons in the AL? To examine subthreshold and spiking responses of follower neurons, we recorded from PNs and LNs in whole-cell current-clamp mode while varying the temperature of the antenna. Cooling the antenna to $6^{\circ} \mathrm{C}$ had two main, reversible effects on PNs. First, consistent with results from our tetrode recordings (Fig. 2), spontaneous and odor-evoked spiking diminished and in most cases ceased completely (Fig. $3 A-E)$. Second, the resting membrane potential of the PNs decreased by $6.9 \pm 0.6 \mathrm{mV}$ (mean \pm SEM, starting from an average of $-54.3 \mathrm{mV}, n=11$ ). Despite the presence of the thermal barrier, in these experiments cooling the antenna also slightly cooled the saline bathing the brain by $<2^{\circ} \mathrm{C}$. To determine whether cooling the brain would affect PN properties, we lowered the temperature of the saline bath $2^{\circ} \mathrm{C}$ by adding drops of chilled saline. Directly cooling the saline bath had no significant effect upon the membrane potential of the PNs (Fig. 3F, open circles). Thus, changes in PN responses were not caused by changes in the temperature of the saline bath.

We also examined LNs, the interneurons within the AL (Fig. $4 A-E$ ). While LNs do not exhibit sodium spikes, their responses when the antenna was cooled were otherwise like those of the PNs: odor-evoked responses in LNs were eliminated and, on av- 

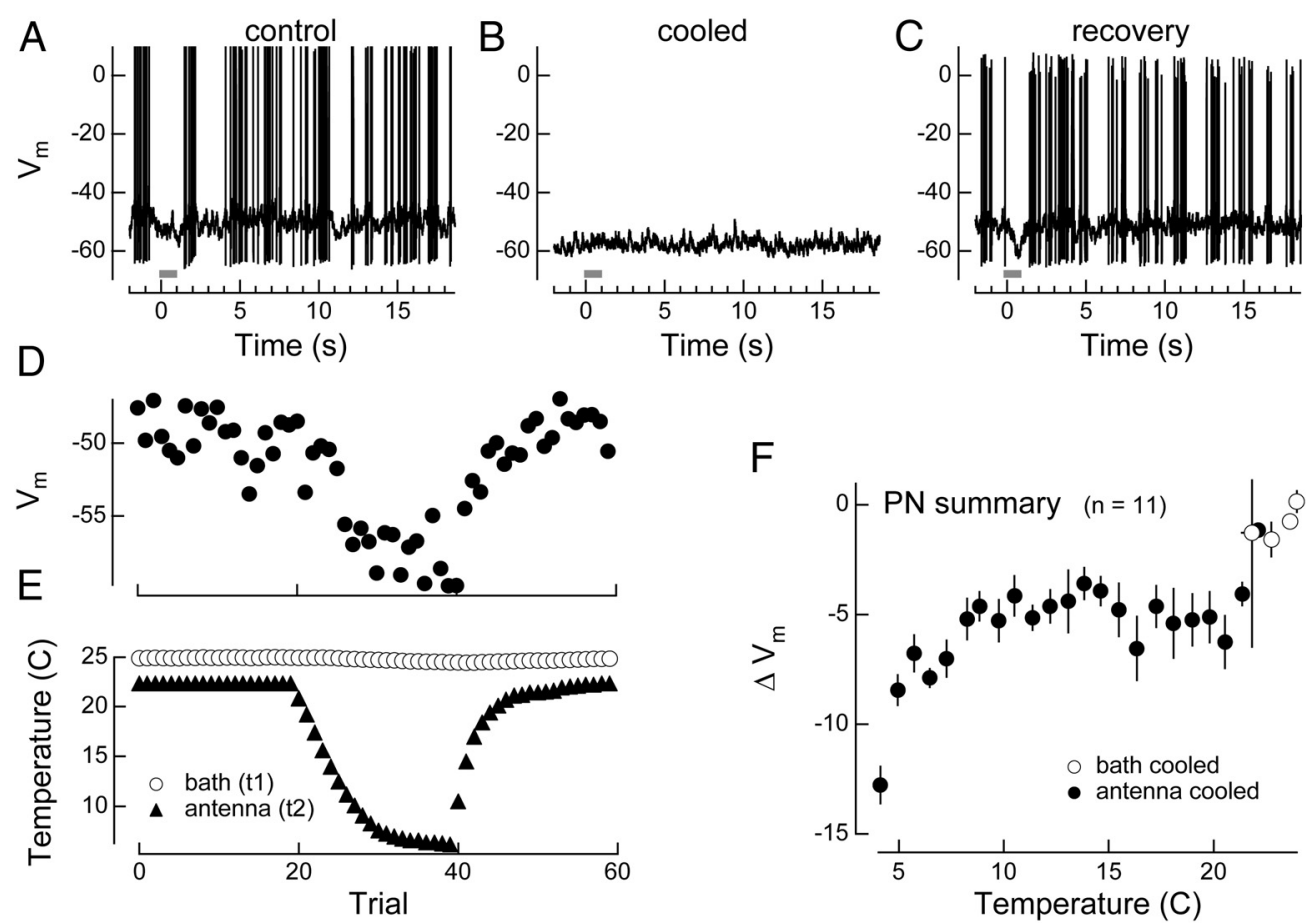

Figure 3. Projection neurons were tonically depolarized by spontaneous activity in ORNs. Cooling the antenna hyperpolarized the resting membrane potential of PNs recorded under whole-cell current-clamp. $\boldsymbol{A}-\boldsymbol{C}$, Response of an example PN to a $1 \mathrm{~s}$ pulse of $100 \%$ hexanol (gray bar) when the antenna was at room temperature $(\boldsymbol{A})$, cooled $(\boldsymbol{B})$, and returned to room temperature (C). $\boldsymbol{D}$, Resting membrane potential (average voltage preceding the odor stimulation), of the same example PN in $\boldsymbol{A}-\boldsymbol{C}$ over the course of the experiment. $\boldsymbol{E}$, Temperature recorded next to the antenna (closed triangles) and next to the brain in the saline bath (open circles) over the course of the experiment. $\boldsymbol{F}$, Summary: average resting membrane potential of all PNs $(n=11)$ when the antenna was cooled (closed circles) and when the saline bath was directly cooled with drops of cold saline to a temperature lower than that caused by cooling the antenna (open circles). Error bars: SEM. Resting membrane potential at room temperature before cooling was $-54.3 \pm 1.1 \mathrm{mV}$ (mean \pm SEM). Membrane potentials were corrected for the junction potential. The change in membrane potential was statistically significant $(p<0.001)$ when the antenna was cooled and not when the bath was directly cooled (see Materials and Methods).
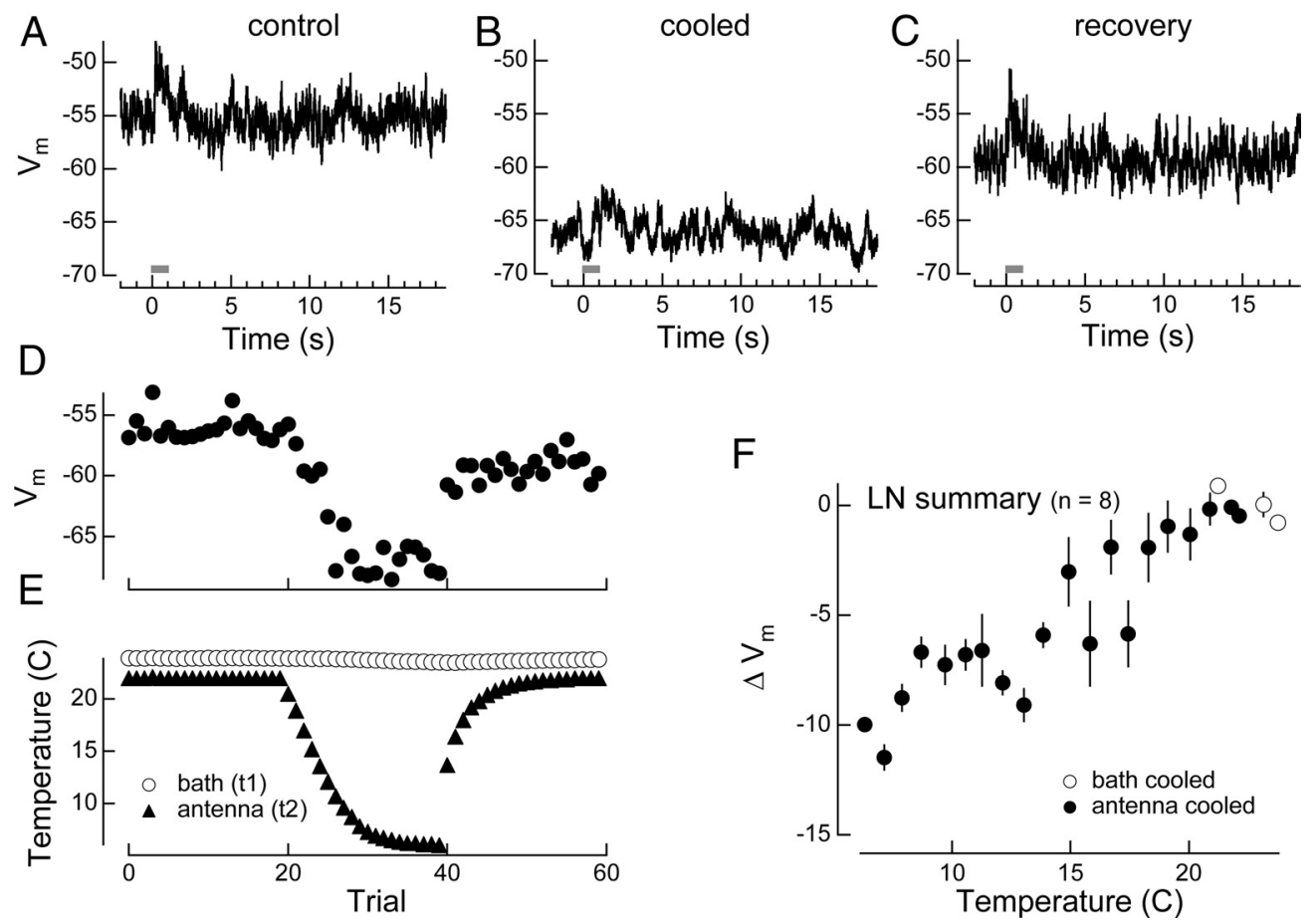

Figure 4. Local neurons were tonically depolarized by spontaneous activity in ORNs. Cooling the antenna hyperpolarized the resting membrane potential of LNs recorded under whole-cell current-clamp. $\boldsymbol{A}-\boldsymbol{E}$, Results from an example LN. All conventions are the same as those described for Figure 3. $\boldsymbol{F}$, Summary: average resting membrane potential of all LNs $(n=8)$. Resting membrane potential at room temperature before cooling was $-58.3 \pm 0.7 \mathrm{mV}$ (mean \pm SEM). Membrane potentials were corrected for the junction potential. The change in membrane potential was statistically significant $(p<0.001)$ when the antenna was cooled and not when the bath was directly cooled (see Materials and Methods). 

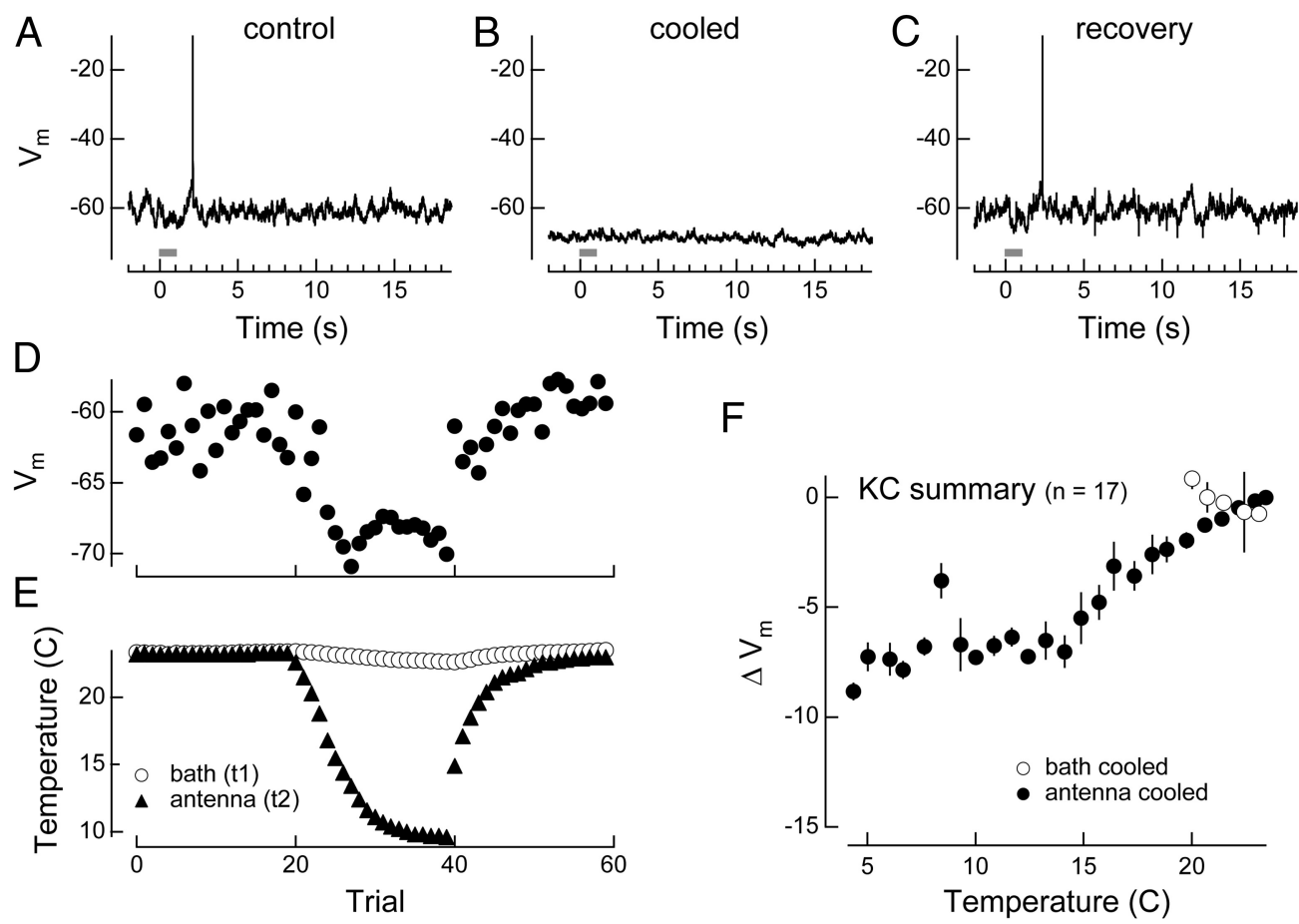

Figure 5. Kenyon cells were tonically depolarized by spontaneous activity in ORNs. Cooling the antenna hyperpolarized the resting membrane potential of KCs recorded under whole-cell current-clamp. $\boldsymbol{A}-\boldsymbol{E}$, Results from an example KC. All conventions are the same as those described for Figure 3. $\boldsymbol{F}$, Summary: average resting membrane potential of all KCs $(n=17)$. Resting membrane potential at room temperature before cooling was $-60.7 \pm 0.6 \mathrm{mV}$ (mean \pm SEM). Membrane potentials were corrected for the junction potential. The change in membrane potential was statistically significant $(p<0.001)$ when the antenna was cooled and not when the bath was directly cooled (see Materials and Methods).

erage, the resting membrane potential decreased by $13.1 \pm 1.0$ $\mathrm{mV}$ (starting from an average of $-58.3 \mathrm{mV}, n=8$ ). As we had observed in PNs, the change in membrane potential was not due to small shifts in the temperature of the saline bath (Fig. $4 F$, open circles).

These results show that the constant barrage of spikes from ORNs caused the PNs and LNs to tonically depolarize even in the absence of deliberate odor stimulation. Despite the significant and tonic depolarization of PNs caused by ORN spikes, PNs did not spike continuously but rather fired at $\sim 2.5$ spikes/s. The modest firing rate of PNs, given the barrage of input they receive, characterizes the spiking threshold of PNs.

What effect does the ongoing excitatory barrage originating in ORNs have further downstream upon the KCs? Compared to PNs, KCs are nearly silent when no odor is applied. Our patch recordings revealed that, as we found in PNs and LNs, cooling the antenna reduced subthreshold baseline activity in KCs and eliminated their spiking responses to odor presentations (Fig. 5A-E). Cooling the antenna caused the KCs to hyperpolarize by $7.4 \pm 0.7$ $\mathrm{mV}$ (starting from an average of $-60.7 \mathrm{mV}, n=17$ ). Directly cooling the saline bath by $2^{\circ} \mathrm{C}$ actually depolarized the resting membrane potential slightly, by $1.6 \mathrm{mV}$ (Fig. $5 F$, open circles).

Together, these results indicate the KCs are tonically depolarized by spikes from PNs that, in turn, are driven by spikes originating in ORNs. Furthermore, variance in the membrane potentials of both $\mathrm{PNs}$ and KCs changed in proportion to the changes in resting membrane potential, as would be expected from a reduction in synaptic input as the antenna is cooled (data not shown).

High firing threshold after maximal convergence of information optimizes odor detection

We found that tonic baseline spiking in ORNs propagates to and tonically elevates the membrane potentials and firing rates of LNs and PNs in the AL, and the resulting baseline spiking in PNs tonically elevates the membrane potentials of KCs. Yet, despite this tonic input, KCs rarely spike; for several reasons (PerezOrive et al., 2002; Demmer and Kloppenburg, 2009; Papadopoulou et al., 2011), KCs have firing thresholds higher than the level of input provided by convergent PNs driven by spontaneous activity in ORNs.

Several lines of evidence suggest that the olfactory system benefits from the sparse representation of odorants in KCs (Laurent, 2002). In principle, a higher response threshold could be set earlier in the olfactory pathway, perhaps in PNs rather than in KCs, leading to greater overall sparseness in the olfactory system. Does the arrangement of different firing thresholds at different stages along the olfactory pathway confer specific advantages for odor encoding? To explore this question we investigated the potential consequences of other possible configurations by simulating the statistics of ORN spiking and its effects upon second and third order neurons in the olfactory pathway (Fig. 6A; see Materials and Methods). In vivo, a given $\mathrm{KC}$ receives input from more types of receptors than does a given $\mathrm{PN}$, allowing $\mathrm{KCs}$ to accurately determine the presence and identity of odors. Reflecting this, our simulation explored parameters including a range of response strength to odors, varying degrees of convergence from ORNs to PNs to KCs, and a range of thresholds for allowing spikes, originating in ORNs, to influence KCs. To determine how combinations of these parameters would affect the abilities of a KC to detect signal amid noise, we simulated trials that included odor-evoked responses (signal) or just spontaneous activity (noise). Then, with a standard receiver operator characteristic model we evaluated how well the $\mathrm{KC}$ could distinguish between signal and noise trials.

Our analysis included a condition that is challenging for signal detection: when the responses of ORNs to an odor were only slightly different from their spontaneous activity. Figure $6 B$ 
shows, for each type of neuron in our model, an example of the cumulative probability of firing rates for varying inputs, given different amounts of spontaneous (blue) or odor-evoked (red) firing in the ORNs. The characteristics of the input to PNs, originating from small subsets of the ORNs, are similar to those of the ORNs: both spontaneous and odorevoked responses largely overlap. But the characteristics of the input to KCs, originating from larger subsets of ORNs by indirect convergence via PNs, are quite different for spontaneous and evoked activity. ROC curves are useful for illustrating signal detection performance under a range of conditions. Figure $6 C$ shows an ROC curve of stimulus detection performance in the $\mathrm{KC}$ simulated in panel Figure $6 \mathrm{~B}$. Here, the diagonal indicates detection at chance; blue dots indicate detection performance for three levels of input (thresholds $a-c$ in Fig. $6 B$ ) to this KC. Because odor-evoked and spontaneous inputs were well separated in this example, the KC's true positive rate exceeded the false positive rate for all three example thresholds. To quantify detection performance in each such test condition, we measured the maximal distance (dashed green line) between a model KC's ROC curve (blue line) and the diagonal line of chance. We used this value of maximal distance as a measure of optimal detection.

To investigate the relationship between signal detection performance of KCs and firing thresholds in PNs, we analyzed ROC curves obtained under a wide range of threshold levels. Figure $6 D$ shows an example of both optimal detection performance (green) and the corresponding baseline PN firing rate (blue) as a function of varying threshold levels between ORNs and the PN. As the PN's firing threshold increased, its rate of spontaneous firing decreased. This example shows the KC's optimal detection performance was high over a broad range of PN thresholds. Notably, though, the KC's signal detection performance precipitately worsened when the $\mathrm{PN}^{\prime}$ s rate of spontaneous activity approached zero.

As shown in Figure 7, we next used the approach illustrated in Figure $6 D$ to examine signal detection in KCs over a broad range of ORN-PN convergence ratios (rows) and ORN response intensities (columns). Signal detection by the KCs was poor when the ORN response to odors was only a small increment above the spontaneous spiking rate, regardless of the convergence ratio (left column, $\delta=$ 0.01 ). Signal detection was best when more ORNs converged upon each PN and when ORNs responded with more spikes to the odor stimulus (bottom right corner of the matrix). Consistent across all these cases is that KC detection was optimal (tan areas in each plot) when the level of spontaneous activity in PNs exceeded zero; that is, when the firing threshold for PNs was set to be relatively low. These simulations demonstrate the relationship between spike threshold levels in PNs and optimal detection performance in KCs: the PN threshold must be low enough that PNs inherit some baseline spik-
B
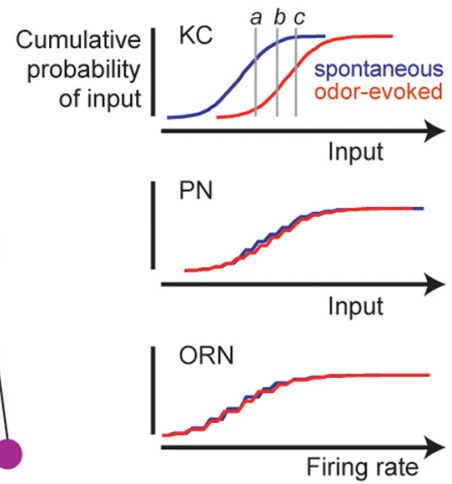

D

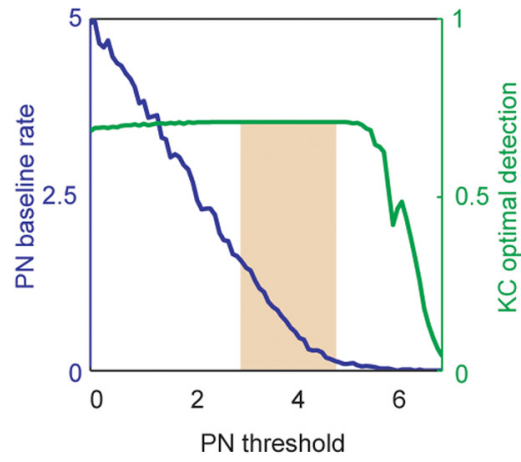

False positive rate

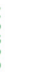 \\ (n)} 응

Figure 6. Simulations show signal detection performance of KCs is best when baseline firing rate of PNs is high. $\boldsymbol{A}$, Schematic illustrates that ORNs converge by receptor type (shown with different colors) onto PNs, which then broadly converge onto KCs. $\boldsymbol{B}$, example threshold levels. $\boldsymbol{C}$, Example receiver operating characteristic curve (blue) shows the computation of a $\mathrm{KC}^{\prime}$ 's varying detection performance for a given PN threshold relative to chance (diagonal) when the threshold of the KC is varied (blue circles $a-c$ correspond to gray lines in B). Green dashed line: a KC's optimal signal detection for a given PN threshold (maximum distance (blue, left axis) as a function of PN threshold. KC detection was optimal (shaded area) for a range of PN thresholds that gave rise to a non-zero PN baseline firing rate. In this example, convergence of ORNs to PN was 10:1.

ing from ORNs, because higher PN thresholds reduce signal along with noise. Thus, given a challenging signal detection task and a background of noisy input, our model suggests that the KCs provide the first location along the olfactory pathway where odor signals can be encoded sparsely, on a near-silent background, without a loss of information.

Our model predicts that raising the firing threshold of PNs to the extent that they no longer fire spontaneously would impair signal detection in the KCs. An observation we subsequently made in vivo is consistent with this prediction. Gradually cooling the antenna hyperpolarizes PNs (Fig. 3), effectively raising the threshold above its rest level. Figure 8 shows that at intermediate temperatures, the spontaneous firing of PNs is silenced while responses to odors persist. With simultaneously recorded pairs of PNs and KCs under these conditions, we found that the odor responses of KCs vanished when the PN threshold was raised. Other factors may also contribute to the elimination of odor-evoked responses in KCs, such as temperaturedependent changes in the temporal patterns of responses evoked by odors in the PNs.

\section{Discussion}

Spontaneous activity in the olfactory system

Abundant spontaneous activity has been observed in ORNs ( Raman et al., 2010; Hallem and Carlson, 2006; Dickens, 1990; 

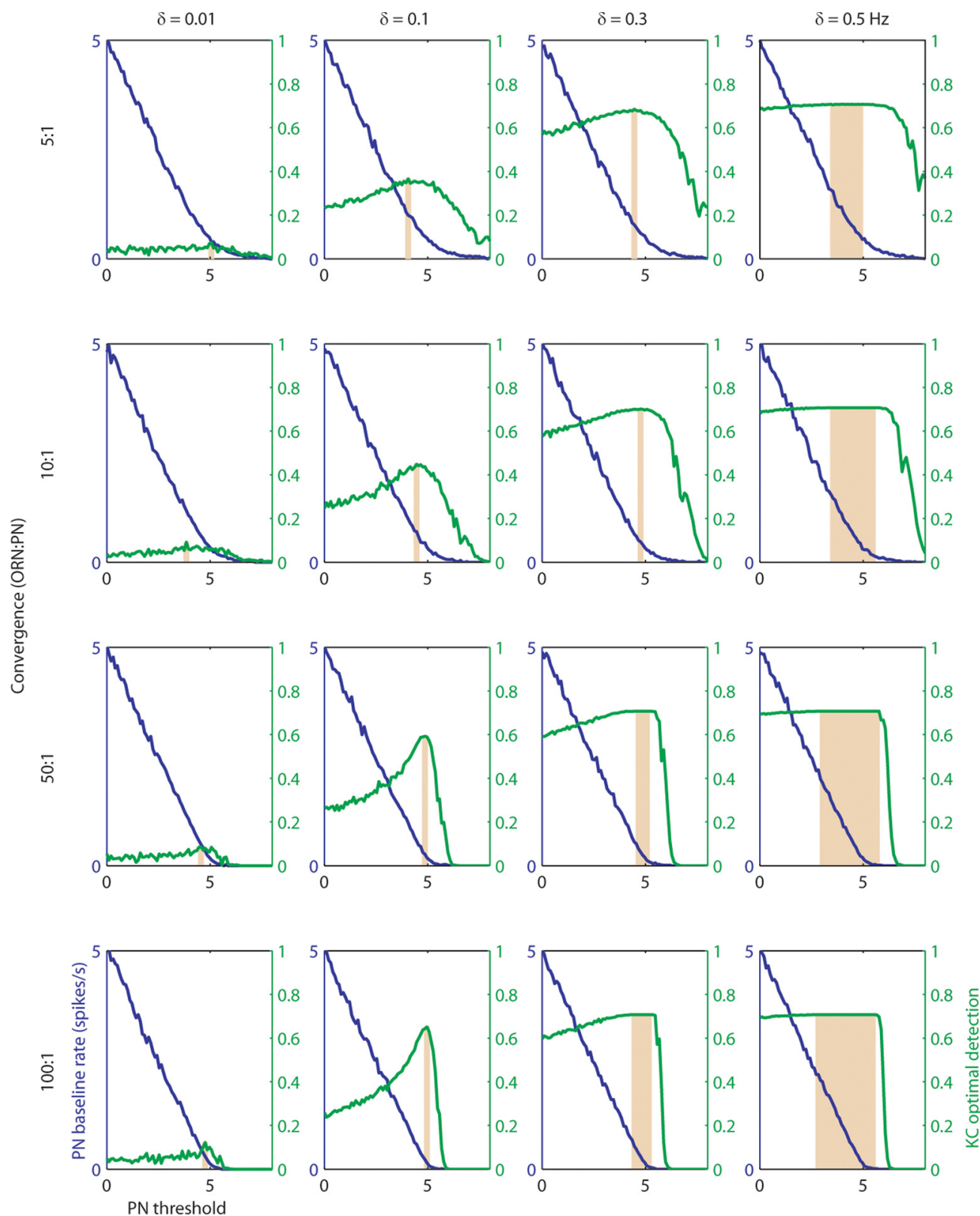

Figure 7. Simulation of Kenyon cell signal detection for a wide range of parameters. Each plot is in the same format as that in Figure $6 \mathrm{D}$ and shows detection performance by $\mathrm{KC}$ ( $\mathrm{green}$, right axis) and PN baseline rate (blue, left axis) as a function of PN threshold level. The convergence ratio of ORNs to PNs was varied (by row, increasing from top to bottom); the mean output of ORNs (spontaneous firing rate of 5 spikes/s plus an odor-evoked rate of $\delta$ was varied (by column, increasing from left to right). KC detection performance was calculated as shown in Fig. 6. Tan regions indicate optimal detection performance for each parameter set. When odors evoked only small changes in the firing rates of ORNs, detection by KCs was poor. When odors evoked greater changes from baseline and when the convergence ratio of ORNs to PNs was greater, detection performance of KCs improved. However, detection performance was never optimal when the baseline firing rate of PNs was zero. Thus, eliminating spontaneous firing in PNs would compromise signal detection in KCs, particularly under challenging conditions (when $\delta$ is low).

Ochieng' and Hansson, 1999; Duchamp-Viret et al., 2000; Rospars et al., 1994) and their immediate neural followers in several species (Philpot et al., 1997; Olsen et al., 2007; Ito et al., 2008). However, neurons located one synapse beyond the immediate followers, such as cortical neurons in mammals (DuchampViret et al., 1996) and KCs in insects (Laurent and Naraghi, 1994; Stopfer et al., 2003; Mazor and Laurent, 2005; Ito et al., 2008; Turner et al., 2008), are often quiet when not responding to presentations of odors.

Our recordings in locusts from ORNs in the antenna, from LNs and PNs of the antennal lobe, and from KCs of the MBs revealed ongoing background activity in the absence of odorant presentations. Cooling the antenna reversibly quieted the ORNs and nearly silenced the PNs, and, consistent with results obtained from Drosophila (Kazama and Wilson, 2009), removing the antenna also silenced the PNs (Figs. 1-3). These results indicate that spontaneous activity in the locust AL does not arise locally within its neurons or from its circuit interactions, but rather is inherited entirely from the ORNs. The constant barrage of spiking originating in ORNs exerts a powerful influence on follower neurons; it tonically and significantly depolarizes the PNs (Fig. 3), LNs (Fig. 4), and KCs (Fig. 5) and is thus a factor in setting the firing thresholds of these neurons. We used a simple model to test the significance of these threshold levels for effective information processing. Our analysis of the receiver operator characteristics of KCs demonstrated that, given the ongoing barrage of activity 
from ORNs and a challenging olfactory task in which signal barely exceeds noise, odor detection is optimal when the firing threshold of PNs is set relatively low to a level that permits some spontaneous spiking. Given the convergence of olfactory neurons, the threshold level between PNs and KCs allows the KCs to respond sparsely, signaling the presence of odors.

\section{Baseline activity in olfactory receptor neurons}

We found that cooling the antenna nearly abolished spontaneous and odor-evoked activity originating in the ORNs. Our study did not address the mechanism by which cooling so affected the ORNs, but contributing factors likely include slowing the motion of odor molecules, decreasing the rates and effectiveness of odorant binding proteins and transduction proteins within the antenna, and changing the intrinsic membrane properties and vesicle release probabilities of the ORNs.

Isolating the ORNs from the environment by coating the antenna with Vaseline reduced spontaneous activity only slightly (Figs. 1,2), suggesting this activity arises largely from spontaneous events occurring within the sensilla rather than from ongoing stimulation by odorants in the environment. Various mechanisms may contribute to the spontaneous firing of sensory neurons; these could include the binding of odorants or their breakdown components lingering in the sensillar lymph, spontaneous isomerizations of the receptor or activation of transduction elements (Rieke and Baylor, 1996, 2000), thermal fluctuations of ion channels (Diba et al., 2004), or spontaneous release of ATP by support cells causing sensory neuron activation (Tritsch et al., 2007).

Our result demonstrating that spontaneous activity in the olfactory system originates in the ORNs is consistent with a previous finding in a vertebrate: naris closure, which isolates ORNs, reduces spiking in mitral cells (Philpot et al., 1997). Furthermore, Drosophila OR83b-null mutants, which lack functional receptors, show no spontaneous activity in the ORNs (Benton et al., 2007), indicating that receptors in ORNs must be active for the neuron to generate baseline spikes. Similarly, Drosophila mutants whose ORNs for pheromone detection lack the olfactory binding protein LUSH also lack spontaneous activity (Benton et al., 2007), indicating that constituents of the sensillar lymph are critical to generating baseline firing.

Why are ORNs so noisy? The prevalence of spontaneous activity in ORNs may reflect general principles and constraints of olfactory transduction. Many ORNs respond to a range of odorsee Results.
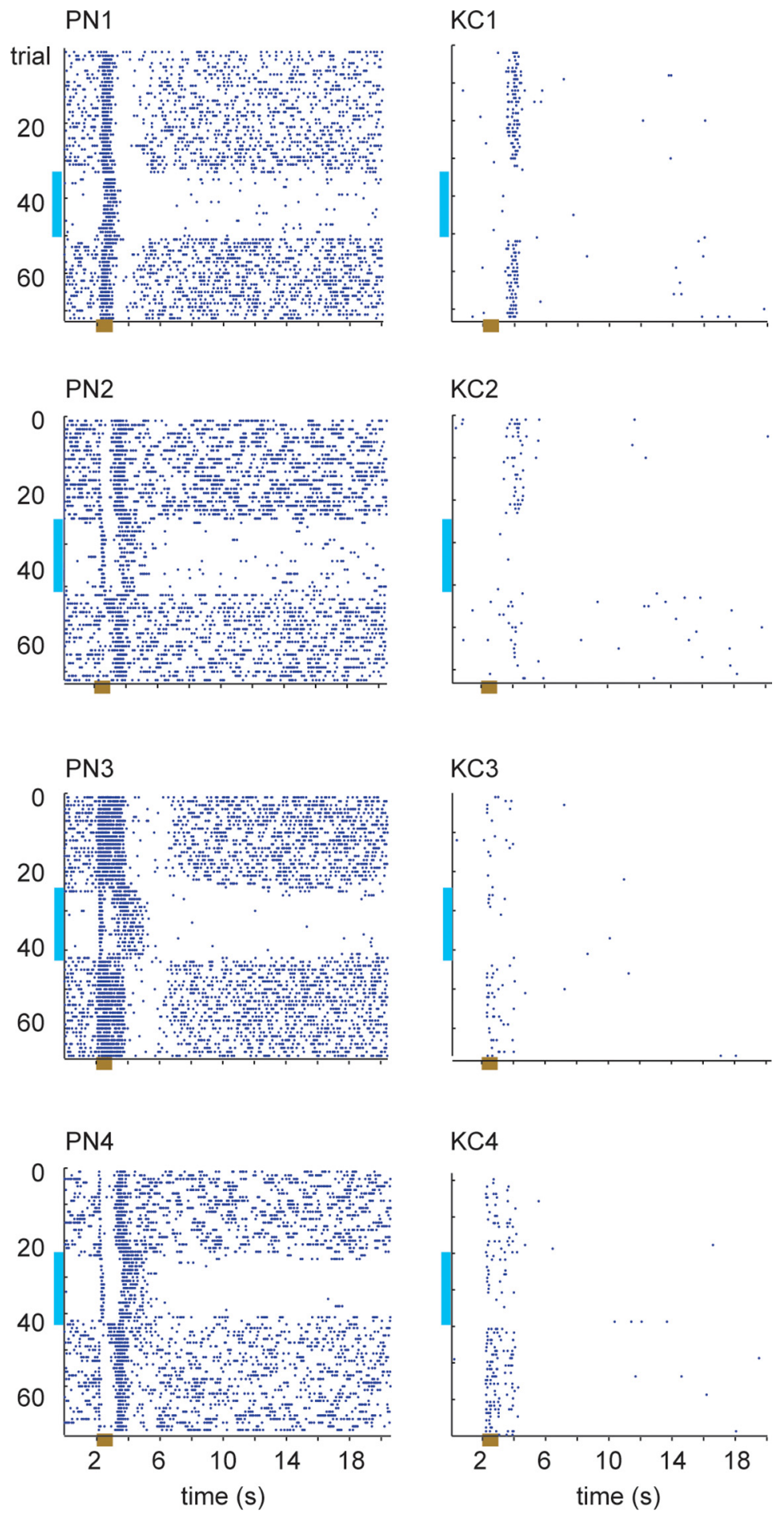

Figure 8. Our model predicts that raising the firing threshold of PNs to the extent that they no longer fire spontaneously would impair signal detection in the KCs. Simultaneously recorded pairs of PNs and KCs show results consistent with this prediction. Cooling the antenna hyperpolarizes PNs (Fig. 3), effectively raising the threshold above its rest level. The odor responses of KCs vanished when the PN threshold was raised. Other factors may also contribute to the elimination of odor-evoked responses in $\mathrm{KCs}$;

ants (Hallem and Carlson, 2006; Ito et al., 2009; Raman et al., 2010). To some extent, ORNs may be activated, in the absence of environmental odorants, by odor binding proteins or other lymph components. Mechanisms for scavenging a diverse assortment of odorants from the sensillar lymph may not operate efficiently for every odorant. These possibilities are consistent with 
our finding that ORNs continued to generate spikes even when the antenna was coated. In general, a transduction mechanism that is highly sensitive to a wide range of odorants will consequently also generate false positives, i.e., noise.

\section{Baseline activity in the antennal lobe and mushroom bodies} The ongoing barrage of activity in ORNs impinges directly upon LNs and PNs, depolarizing their membrane potentials and thus bringing them closer to their thresholds for firing. How ongoing activity may contribute to information processing in the AL is not known. One possibility is that ongoing noisy input may define an elevated background against which periods of inhibition can have an effect, thus expanding the dynamic range in which inhibitory LNs can contribute to the temporal patterning of odor codes observed in PNs. Another possibility is that spontaneous activity may in some way help maintain the dynamical regime of activity within AL circuitry required for PNs to respond rapidly to odor presentations. In cortical neurons, fluctuating stimuli have been shown to help enable precise spike timing (Mainen and Sejnowski, 1995). However, the fluctuations in the membrane potentials of PNs introduced by baseline activity in ORNs are not repeatable, making it unlikely these fluctuations could contribute to the reliability or precision of olfactory responses.

Background spiking in the AL may simply carry over from biophysical constraints on the highly sensitive ORNs and provide no benefits at all. Such activity could, in fact, only serve to obscure odor signals and waste metabolic energy required for spiking. Could neurons of the AL mitigate these negative consequences by setting thresholds appropriate to screen out most background activity, as the KCs appear to do? PNs do, of course, display effective spike thresholds-despite the significant, ongoing depolarization by $\sim 7 \mathrm{mV}$ in PNs originating with the tonic input from ORNs (Fig. 3), PNs at rest spike only at $\sim 2.5$ spikes/s; thus, for stretches of time averaging $500 \mathrm{~ms}$, PNs are depolarized by ORNs yet do not spike. Why not mitigate the potential disadvantages of background activity by raising response thresholds of PNs even higher? Our results show that reducing background spiking in the AL by raising response thresholds would likely reduce some odor-evoked spiking as well, reducing the information content of the spike trains. Indeed, the results of our simulation suggest that raising the response thresholds of PNs would diminish the detection capabilities of KCs (Fig. 6).

Why is this so? In animals where a precision analysis of the convergence of ORNs to PNs is possible, it has been shown that ORNs converge by receptor type onto PNs (Mombaerts et al., 1996; Vosshall et al., 2000). Over a range of concentrations, any given odorant can activate a diverse assortment of ORNs (Hallem and Carlson, 2006; Ito et al., 2009). Therefore, PNs can respond to and carry information about multiple odorants (Stopfer et al., 2003; Wilson et al. 2004; Mazor and Laurent, 2005) so that information about odorants is broadly distributed across many PNs. The responses of any given PN will contain ambiguity about the odorant eliciting the response. The PN to KC synapse represents the first convergence site for information arising from a wide variety of ORN types. In locusts, large numbers of PNs connect to any given KC (Jortner et al., 2007). Thus, along the olfactory pathway, the first place this ambiguity about odor stimuli can be resolved is in the KCs. Because the mean baseline rate of firing is relatively constant across PNs (Stopfer et al., 2003; Mazor and Laurent, 2005), KCs are well positioned to set a uniformly high threshold, allowing them to sparsely signal their relatively unambiguous identifications of odors. Given the background output of ORNs and the convergence patterns of ORNs, PNs, and KCs, our simulation of a challenging olfactory task (Fig. 6) indicates that the firing thresholds of PNs and KCs are set to maximize signal detection in KCs, the first olfactory neurons positioned to report information about the odor sparsely.

Our exploration of noise sources in locust olfaction provides a specific example of how a detection system, bombarded by noise at the first stage of processing, balances the competing challenges of maintaining sensitivity to a wide range of stimuli and setting thresholds to eliminate noise and to generate sparse internal sensory representations. These principles may apply to other natural or artificial sensory systems that employ multiple stages of processing and circuitry convergence to achieve optimal detection.

\section{References}

Benton R, Vannice KS, Vosshall LB (2007) An essential role for a CD36related receptor in pheromone detection in Drosophila. Nature 450:289-293.

Brecht M, Schneider M, Sakmann B, Margrie TW (2004) Whisker movements evoked by stimulation of single pyramidal cells in rat motor cortex. Nature 427:704-710.

Dave AS, Margoliash D (2000) Song replay during sleep and computational rules for sensorimotor vocal learning. Science 290:812-816.

Demmer H, Kloppenburg P (2009) Intrinsic membrane properties and inhibitory synaptic input of Kenyon cells as mechanisms for sparse coding? J Neurophysiol 102:1538-1550.

DeWeese MR, Wehr M, Zador AM (2003) Binary spiking in auditory cortex. J Neurosci 23:7940-7949.

Diba K, Lester HA, Koch C (2004) Intrinsic noise in cultured hippocampal neurons: experiment and modeling. J Neurosci 24:9723-9733.

Dickens JC (1990) Specialized receptor neurons for pheromones and host plant odors in the boll weevil, Anthonomus grandis Boh. (Coleoptera: Curculionidae). Chem Senses 15:311-331.

Drake KL, Wise KD, Farraye J, Anderson DJ, BeMent SL (1988) Performance of planar multisite microprobes in recording extra-cellular singleunit intracortical activity. IEEE Trans Biomed Eng 35:719-732.

Duchamp-Viret P, Palouzier-Paulignan B, Duchamp A (1996) Odor coding properties of frog olfactory cortical neurons. Neuroscience 74:885-895.

Duchamp-Viret P, Duchamp A, Chaput MA (2000) Peripheral odor coding in the rat and frog: quality and intensity specification. J Neurosci 20:2383-2390.

Euston DR, Tatsuno M, McNaughton BL (2007) Fast-forward playback of recent memory sequences in prefrontal cortex during sleep. Science 318:1147-1150

Griffin JD, Boulant JA (1995) Temperature effects on membrane potential and input resistance in rat hypothalamic neurons. J Physiol 488:407-418.

Hahnloser RH, Kozhevnikov AA, Fee MS (2002) An ultra-sparse code underlies the generation of neural sequences in a songbird. Nature 419:65-70.

Hallem EA, Carlson JR (2006) Coding of odors by a receptor repertoire. Cell 125:143-160.

Ito I, Ong RC, Raman B, Stopfer M (2008) Sparse odor representation and olfactory learning. Nat Neurosci 11:1177-1184.

Ito I, Bazhenov M, Ong RC, Raman B, Stopfer M (2009) Frequency transitions in odor-evoked neural oscillations. Neuron 64:692-706.

Jefferis GS, Vyas RM, Berdnik D, Ramaekers A, Stocker RF, Tanaka NK, Ito K, Luo L (2004) Developmental origin of wiring specificity in the olfactory system of Drosophila. Development 131:117-130.

Jortner RA, Farivar SS, Laurent G (2007) A simple connectivity scheme for sparse coding in an olfactory system. J Neurosci 27:1659-1669.

Kazama H, Wilson RI (2009) Origins of correlated activity in an olfactory circuit. Nat Neurosci 12:1136-1144.

Kenet T, Bibitchkov D, Tsodyks M, Grinvald A, Arieli A (2003) Spontaneously emerging cortical representations of visual attributes. Nature 425:954-956.

Laurent G (2002) Olfactory network dynamics and the coding of multidimensional signals. Nat Rev Neurosci 3:884-895.

Laurent G, Davidowitz H (1994) Encoding of olfactory information with oscillating neural assemblies. Science 265:1872-1875.

Laurent G, Naraghi M (1994) Odorant-induced oscillations in the mushroom bodies of the locust. J Neurosci 14:2993-3004. 
Limb CJ, Braun AR (2008) Neural substrates of spontaneous musical performance: an FMRI study of jazz improvisation. PLoS One 3:e1679.

MacLean JN, Watson BO, Aaron GB, Yuste R (2005) Internal dynamics determine the cortical response to thalamic stimulation. Neuron 48:811-823.

Mainen ZF, Sejnowski TJ (1995) Reliability of spike timing in neocortical neurons. Science 268:1503-1506.

Maye A, Hsieh CH, Sugihara G, Brembs B (2007) Order in spontaneous behavior. PLoS One 2:e443.

Mazor O, Laurent G (2005) Transient dynamics versus fixed points in odor representations by locust antennal lobe projection neurons. Neuron 48:661-673.

Mombaerts P, Wang F, Dulac C, Chao SK, Nemes A, Mendelsohn M, Edmondson J, Axel R (1996) Visualizing an olfactory sensory map. Cell 87:675-686.

Ochieng' SA, Hansson BS (1999) Responses of olfactory receptor neurones to behaviourally important odours in gregarious and solitarious desert locust, Schistocerca gregaria. Physiol Entomol 24:28-36.

Olsen SR, Bhandawat V, Wilson RI (2007) Excitatory interactions between olfactory processing channels in the Drosophila antennal lobe. Neuron 54:89-103.

Papadopoulou M, Cassenaer S, Nowotny T, Laurent G (2011) Normalization for sparse encoding of odors by a wide-field interneuron. Science 332:721-725.

Perez-Orive J, Mazor O, Turner GC, Cassenaer S, Wilson RI, Laurent G (2002) Oscillations and sparsening of odor representations in the mushroom body. Science 297:359-365.

Perez-Orive J, Bazhenov M, Laurent G (2004) Intrinsic and circuit properties favor coincidence detection for decoding oscillatory input. J Neurosci 24:6037-6047.

Philpot BD, Foster TC, Brunjes PC (1997) Mitral/tufted cell activity is attenuated and becomes uncoupled from respiration following naris closure. J Neurobiol 33:374-386.
Pouzat C, Mazor O, Laurent G (2002) Using noise signature to optimize spike-sorting and to assess neuronal classification quality. J Neurosci Methods 122:43-57.

Raman B, Joseph J, Tang J, Stopfer M (2010) Temporally diverse firing patterns in olfactory receptor neurons underlie spatiotemporal neural codes for odors. J Neurosci 30:1994-2006.

Rieke F, Baylor DA (1996) Molecular origin of continuous dark noise in rod photoreceptors. Biophys J 71:2553-2572.

Rieke F, Baylor DA (2000) Origin and functional impact of dark noise in retinal cones. Neuron 26:181-186.

Rospars JP, Lánský P, Vaillant J, Duchamp-Viret P, Duchamp A (1994) Spontaneous activity of first- and second-order neurons in the frog olfactory system. Brain Res 662:31-44.

Stopfer M, Jayaraman V, Laurent G (2003) Intensity versus identity coding in an olfactory system. Neuron 39:991-1004.

Tritsch NX, Yi E, Gale JE, Glowatzki E, Bergles DE (2007) The origin of spontaneous activity in the developing auditory system. Nature 450:50-55.

Turner GC, Bazhenov M, Laurent G (2008) Olfactory representations by Drosophila mushroom body neurons. J Neurophysiol 99:734-746.

Vosshall LB, Wong AM, Axel R (2000) An olfactory sensory map in the fly brain. Cell 102:147-159.

Wilson RI, Turner GC, Laurent G (2004) Transformation of olfactory representations in the Drosophila antennal lobe. Science 303:366-370.

Young MP, Yamane S (1992) Sparse population coding of faces in the inferotemporal cortex. Science 256:1327-1331.

Yu CR, Power J, Barnea G, O'Donnell S, Brown HE, Osborne J, Axel R, Gogos JA (2004) Spontaneous neural activity is required for the establishment and maintenance of the olfactory sensory map. Neuron 42:553-566. 